\title{
THE PROJECTIVE DIMENSION OF A COMPACT ABELIAN GROUP
}

\author{
ERIC C. NUMMELA
}

\begin{abstract}
Let $X$ be a compact group, $F G X$ the (Graev) free topological group generated by $X$, and $K$ the kernel of the canonical quotient morphism from $F G X$ to $X$. Then $K$ is a (Graev) free topological group. A corollary to the abelian analogue of this theorem is that the projective dimension of a compact abelian group, relative to the class of all continuous epimorphisms admitting sections, is exactly one.
\end{abstract}

0 . Introduction. Let $\mathscr{G}$ (respectively, $\mathscr{A}$ ) denote the category of topological (abelian) groups and continuous group homomorphisms. We take as known the definitions and elementary properties of topological groups as presented in, for example, [2, pp. 219-250]. We adopt the convention that all groups are topological, using the modifier "algebraic" for those groups without topologies. Moreover, all our topologies satisfy the Hausdorff separation axiom.

We assume also some familiarity with the notions of free (topological) groups due to A. A. Markov [8] and M. I. Graev [5]. Given a completely regular space $X$, we denote by $F X(Z X)$ the Markov free (free abelian) group over $X$, and by $F G X(Z G X)$ the Graev free (free abelian) group over $X$. Recall that if $X$ is a compact space, then $F G X$ is the colimit of the expanding sequence $\left\{F_{m}\right\}$ of compact spaces, where $F_{m}$ is the set of all words in $F G X$ whose reduced length relative to $X \backslash\{e\}$ is less than or equal to $m$ ( $e$ is the identity of $F G X$ ). An analogous result holds for $F X, Z G X$, and $Z X$.

Recall that a group $P$ in $\mathscr{G}(\mathscr{A})$ is projective relative to a class $\mathscr{E}$ of epimorphisms in $\mathscr{G}(\mathscr{A})$ if, given any diagram

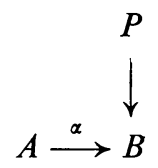

Received by the editors February 25, 1972 and, in revised form, August 18, 1972. AMS (MOS) subject classifications (1970). Primary 18G05, 18G20, 18G25, 22C05; Secondary 13D05, 18G10, 18G15, 20E05, $22 \mathrm{~A} 99$.

Key words and phrases. Markov free topological group, Graev free topological group, projective abelian group, Schreier system.

(c) American Mathematical Society 1973 
in $\mathscr{G}(\mathscr{A})$, with $\alpha \in \mathscr{E}$, there is a morphism $P \rightarrow A$ in $\mathscr{G}(\mathscr{A})$ making the diagram commute. C. E. Hall [6] has shown that the class $\mathscr{T}_{e}\left(\mathscr{S}_{e}\right)$ of all epimorphisms in $\mathscr{G}(\mathscr{A})$ which admit sections (i.e., continuous right inverse functions) is the largest class of epimorphisms relative to which Markov free (free abelian) groups are projective. F. R. Ward [12] announced that Graev free abelian groups are also projective relative to $\mathscr{S}_{e}$; indeed, it is shown in [10] that the Graev free (free abelian) groups also determine the class $\mathscr{T}_{e}\left(\mathscr{S}_{\mathrm{p}}\right)$. Thus when we say "projective", we will mean "projective relative to $\mathscr{T}_{e}\left(\mathscr{S}_{e}\right)$ ".

In a forthcoming paper [10], we characterize the class $\mathscr{S}$ of short exact sequences in $\mathscr{A}$ determined by $\mathscr{S}_{e}$. (In fact, this is done in the category of topological $R$-modules over any topological ring $R$ with identity.) We show that the class $\mathscr{S}$ is proper, hence suitable for constructing a (relative) bifunctor Ext* with values in the category of algebraic abelian groups. Moreover, $\operatorname{Ext}^{*}(B, A)$ can be computed via any projective resolution of $B$, and thus the concept of projective dimension is meaningful in this context.

The reader may wish to contrast our work with that of M. Moskowitz in the category $\mathscr{L}$ of locally compact abelian groups [9]. J. Dixmier [3] and P. R. Ahern and R. I. Jewett [1] independently characterized the injectives in $\mathscr{L}$ as groups of the form $R^{n} \times T^{\sigma}$, where $R$ is the additive group of real numbers, $T$ is the circle group, $n$ is finite, and $\sigma$ is an arbitrary cardinal number. This characterization was derived independently by Moskowitz, who used Pontryagin duality theory to characterize the projectives in $\mathscr{L}$ relative to all quotient morphisms. Thus projectives are of the form $R^{n} \times Z^{(\sigma)}$, where $Z^{(\sigma)}$ is a discrete free abelian group of rank $\sigma$. He also proved that a group in $\mathscr{L}$ has a projective resolution if and only if the group is without small subgroups, in which case the resolution is short [9, Theorem 3.6(2)]. That is, in $\mathscr{L}$ the concept of projective dimension is meaningful only for groups without small subgroups, and such groups have projective dimension less than or equal to one.

1. Free subgroups of free groups. We restrict our attention now to free (free abelian) groups as defined by Graev. It is well known that subgroups of free groups need not be free. In fact, Graev gives an example of a closed subgroup of $Z G X$, for $X$ a countable compact space, which is not free [5, pp. 358-364]. However, there is a partial remedy.

(1.1) Definition [5, p. 356]. Let $Y$ be a subset of $F G X$ for some space $X$. Then $Y$ is regularly situated with respect to $X$ if, in the subgroup $\langle Y\rangle \subseteq F G X$ algebraically generated by $Y$, any sequence of words with lengths bounded with respect to $X \backslash\{e\}$ also have lengths bounded with respect to $Y \backslash\{e\}$ ( $e$ is the identity of $F G X)$.

(1.2) Proposition [5, p. 356]. Let $X$ be compact, $Y \subseteq F G X, Y$ compact, 
$e \in Y$, and $Y \backslash\{e\}$ algebraically a free set of generators for $\langle Y\rangle$. Then $\langle Y\rangle$ is closed in FGX and free over $Y$ if and only if $Y$ is regularly situated.

O. Schreier's original proof that subgroups of algebraic free groups are free [11] used the following concepts, given here as detailed by $\mathbf{M}$. Hall, Jr. [7].

(1.3) Definition [7, p. 94]. A set $S$ contained in the algebraic free group over the set $X$ is a (two-sided) Schreier system if for each $\alpha \in S$ :

(1) $\alpha=x_{1} \cdot x_{2} \cdot \ldots \cdot x_{n}$ is reduced as written (each $x_{i} \in X \cup X^{-1}$ ),

(2) $x_{1} \cdot x_{2} \cdot \ldots \cdot x_{n-1} \in S$,

(3) $x_{2} \cdot \ldots \cdot x_{n} \in S$.

Note that a Schreier system always contains $e$.

(1.4) Remark. The concept of "regularly situated" (and Graev's resulting theorems) is easily extended to Markov free groups. However, $X \subseteq F G X$ is a Schreier system, whereas $X \subseteq F X$ is not.

The following lemma shows the utility of Schreier systems.

(1.5) Lemma. Let $K$ be a normal subgroup of the algebraic free group $A$ on the set $X$, and let $\psi: A \rightarrow A \mid K$ be the quotient morphism.

(1) It is possible to choose a complete system of representatives of cosets of $K$ which forms a Schreier system $S$ [7, Lemma 7.2, p. 95]. Let $i: A \mid K \rightarrow A$ map cosets to their representatives in $S$.

(2) Let $B=\left\{s \cdot x \cdot i \psi(s \cdot x)^{-1} \mid s \in S, x \in X\right\}$. Then $B \backslash\{e\}$, where $e$ is the identity of $A$, is a free set of generators for $K$ [7, Lemma 7.2.6, p. 98].

Combining the work of Schreier and Graev, we obtain the following proposition.

(1.6) Proposition. Let $X$ be a compact group (written multiplicatively). Let $\psi: F G X \rightarrow X$ be the canonical quotient morphism, and let $\sigma: S X \rightarrow F G X$ be the kernel of $\psi$. Then $S X$ is free in the sense of Graev.

Proof. We know that $S X$ is a closed normal subgroup of $F G X$, and the elements of $X$, considered as elements of $F G X$, form a Schreier system relative to $S X$. Algebraically, $F G X$ is the free group on the set $X \backslash\{e\}$, where $e$ is the identity of $X$. Thus

$$
B=\left\{x \cdot z \cdot \psi(x \cdot z)^{-1} \mid x \in X, z \in X \backslash\{e\}\right\} \subseteq F G X
$$

algebraically generates $S X$. Now

$$
\psi(x \cdot z)=x z \quad \text { (multiplication in } X)
$$

for all $x, z \in X$. Hence

$$
\begin{aligned}
B & =\left\{x \cdot z \cdot(x z)^{-1} \mid x \in X, z \in X \backslash\{e\}\right\} \\
& =\left\{x \cdot z \cdot(x z)^{-1} \mid x \in X, z \in X\right\} .
\end{aligned}
$$


Since $X$ is compact, $B$ is also compact. Thus we need only show that $B$ is regularly situated to complete the proof. It is an easy exercise to show that if the length of a word in $S X$ relative to $X \backslash\{e\}$ is $\leqq 3$, then its length relative to $B \backslash\{e\}$ is also $\leqq 3$. We outline a simple inductive proof which shows that a word in $S X$ of length $\leqq n$ (where $n \geqq 4$ ) has length $\leqq 3(n-2)$ relative to $B \backslash\{e\}$.

Let $\alpha=x_{1} \cdot x_{2} \cdot x_{3} \cdot x_{4}$ be a reduced word in $S X$ of length 4 (so each $\left.x_{i} \in X \cup X^{-1}\right)$. Then

$$
\alpha=x_{1} \cdot x_{2} \cdot x_{3} \cdot x_{4}=\left[x_{1} \cdot x_{2} \cdot \psi\left(x_{3} \cdot x_{4}\right)\right] \cdot\left[\psi\left(x_{3} \cdot x_{4}\right)^{-1} \cdot x_{3} \cdot x_{4}\right] .
$$

Since $\psi(X) \subseteq X$, each word in brackets is in $S X$. Thus the length of $\propto$ relative to $B \backslash\{e\}$ is $\leqq 3 \cdot 2=3(4-2)$.

The remainder of the proof is omitted.

(1.7) RemarK. It seems likely that the above proposition is also true with $F G X$ replaced by $F X$ and "Graev" replaced by "Markov". However, finding a compact, regularly situated Schreier system in $F X$ may be quite difficult. ${ }^{1}$

The following proposition is the abelian analogue of Proposition (1.6), and has an analogous proof.

(1.8) Proposition. Let $A$ be a compact abelian group, and let $0 \rightarrow$ $T A \rightarrow Z G A \rightarrow A \rightarrow 0$ be the canonical short exact sequence involving the Graev free abelian group over A. Then TA is a Graev free abelian group.

\section{The projective dimension of a compact abelian group.}

(2.1) LeMMA. A locally compact (abelian) group is projective if and only if it is a discrete free (free abelian) group. In particular, there are no compact projective (abelian) groups.

Proof. We prove this in $\mathscr{G}$. Suppose $P$ is a projective group. Then there is a morphism $p: P \rightarrow F G P$ in $\mathscr{G}$ such that the triangle

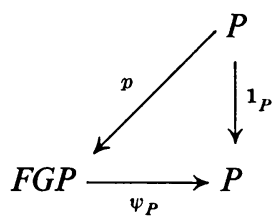

is commutative. Thus $P$ is algebraically isomorphic to a subgroup of a free group, and is hence algebraically a free group. But R. M. Dudley has

\footnotetext{
${ }^{1} \mathrm{~S}$. A. Morris in Varieties of topological groups and left adjoint functors, J. Austral. Math. Soc. (to appear) has proved that Proposition (1.6) is, indeed, true with "Graev" replaced by "Markov".
} 
proved that the only locally compact group topology admitted by an algebraic free (free abelian) group is the discrete topology [4, Corollary 2, p. 589]. (The author is indebted to the referee for pointing out this reference.)

(2.2) THEOREM. Let A be a compact abelian group. Then the projective dimension $d(A)$ is exactly one.

Proof. Proposition (1.8), together with the "independence of projective resolution" theorem of [10], shows that $d(A) \leqq 1$. Lemma (2.1) shows that $d(A) \geqq 1$. Hence $d(A)=1$.

We end this note with the natural question: Is the projective dimension of every (topological) abelian group less than or equal to one? Just for the record, the author conjectures that the answer to this question is "no".

\section{REFERENCES}

1. P. R. Ahern and R. I. Jewett, Factorization of locally compact abelian groups, Illinois J. Math. 9 (1965), 230-235. MR 31 \#3536.

2. N. Bourbaki, Éléments de mathématique. Part. 1. Les structures fondamentales de l'analyse. Livre III: Topologie générale, Hermann, Paris; Addison-Wesley, Reading, Mass., 1966. MR 34 \#5044b.

3. J. Dixmier, Quelques propriétés des groupes abéliens localement compacts, Bull. Sci. Math. (2) 81 (1957), 38-48. MR 20 \#3926.

4. R. M. Dudley, Continuity of homomorphisms, Duke Math. J. 28 (1961), 587-594. MR 25 \#141.

5. M. I. Graev, Free topological groups, Izv. Akad. Nauk SSSR Ser. Mat. 12 (1948), 279-324; English transl. Amer. Math. Soc. Transl. (1) 8 (1962), 305-364. MR 10, 11.

6. C. E. Hall, Projective topological groups, Proc. Amer. Math. Soc. 18 (1967), 425-431. MR 35 \#2994.

7. M. Hall, Jr., Theory of groups, Macmillan, New York, 1959. MR 21 \#1996.

8. A. A. Markov, On free topological groups, Izv. Akad. Nauk SSSR Ser. Mat. 9 (1945), 3-64; English transl., Amer. Math. Soc. Transl. (1) 8 (1962), 195-272. MR 7, 7.

9. M. Moskowitz, Homological algebra in locally compact abelian groups, Trans. Amer. Math. Soc. 127 (1967), 361-404. MR 35 \#5861.

10. E. C. Nummela, Homological algebra of topological modules, Duke Math. J. (submitted).

11. O. Schreier, Die Untergruppen der freien Gruppen, Abh. Math. Sem. Univ. Hamburg. 5 (1927), 161-183.

12. F. R. Ward, On free and projective topological groups, Notices Amer. Math. Soc. 17 (1970), 135. Abstract \#672-182.

Department of Mathematics, University of Florida, Gainesville, Florida 32601 